REVISTA CATALANA DE DRET AMBIENTAL Vol. VI Núm. 1 (2015): 1 - 5

-Crònica-

DERECHO Y POLÍTICAS AMBIENTALES EN NAVARRA

\author{
José Francisco AlENZA García \\ Profesor titular de Derecho Administrativo \\ Universidad Pública de Navarra
}


Sumario: 1. Panorama general. 2. El "desarrollo" del Reglamento de desarrollo de la Ley Foral de Intervención para la Protección Ambiental. 3. La regulación de impuestos energéticos ambientales. 4. Relación de disposiciones.

\section{Panorama general}

Dos novedades de interés presenta el paisaje legislativo navarro de naturaleza ambiental en este primer semestre de 2015. En primer lugar, la Orden Foral de desarrollo del Reglamento de la Ley Foral de Intervención para la Protección Ambiental. En segundo lugar, la regulación legal de dos impuestos energéticos que tienen entre sus objetivos la protección ambiental y la lucha contra el cambio climático. A estas dos disposiciones les dedicaré una atención especial.

Por lo demás, cabe reseñar que ha continuado el proceso de configuración de la red Natura 2000 con la designación y regulación mediante sendos decretos forales de dos nuevas zonas de especial conservación (Belate y Sierra de Aralar).

También se han aprobado otras disposiciones que anualmente se ocupan de diversas materias. En este semestre han sido tres: el Programa de Inspección Ambiental de la Actividad Industrial (Orden Foral 82/2015, de 6 de marzo); las normas de condicionalidad que deben cumplir los beneficiarios que reciban las ayudas económicas de los programas de apoyo al sector vitivinícola (Orden Foral 110/2015, de 13 de abril); y la normativa sobre la pesca del año 2015, que incluye determinadas medidas de control de poblaciones de especies exóticas invasoras (Orden Foral 42/2015, de 10 de febrero).

Finalmente, también cabe señalar que se han modificado el pliego de condiciones y el formulario técnico para la elaboración de los planes de ordenación cinegética en Navarra en virtud de la Resolución 211/2015, del Director General de Medio Ambiente y Agua.

\section{El "desarrollo" del Reglamento de desarrollo de la Ley Foral de Intervención para la Protección Ambiental}

Mediante una orden foral (la 448/2014, de 23 de diciembre) se han aprobado normas de desarrollo del Decreto Foral 93/2006, de 28 de diciembre, por el que se aprueba el reglamento de desarrollo de la Ley Foral 4/2005, de 22 de marzo, de Intervención para 
la Protección Ambiental (en adelante, LFIPA), con el fin de incorporar medidas de agilización administrativa y simplificación procedimental.

Lo que aparentemente se presenta como un desarrollo de un reglamento de desarrollo de la LFIPA para la simplificación procedimental incluye algunos aspectos novedosos y, en ocasiones, de hasta dudosa legalidad, ya que impone algunas obligaciones nuevas (o bien amplía las potestades administrativas de intervención y control) sin una clara cobertura legal.

Se trata de un texto extenso de 34 artículos cuyas novedades alcanzan a las distintas autorizaciones, licencias y formas de intervención ambiental:

- Autorización ambiental integrada. Se introducen modificaciones o precisiones en relación con la remisión del estudio de impacto ambiental, la presentación telemática de la solicitud y la documentación técnica que debe presentarse junto con la solicitud. Se establecen nuevos deberes de información y se amplía la potestad de la Administración de revisión de la autorización ambiental integrada. Se precisan otros aspectos sobre las modificaciones de las instalaciones y la caducidad de la autorización. Se establece el deber de presentar una comunicación previa al cese temporal de las actividades y se regula ex novo el procedimiento que debe seguirse para la autorización del cierre definitivo de las instalaciones, que incluye la presentación de un proyecto técnico de cierre y una evaluación del estado del suelo y de las aguas subterráneas. La resolución del procedimiento de cierre de la instalación podrá incluir la exigencia al titular de la constitución de una fianza económica que responda de los costes inherentes al cierre de la instalación.

- La regulación de la autorización de afectaciones ambientales está mínimamente desarrollada, ocupándose la Orden Foral tan solo de su presentación telemática y de su caducidad.

- La autorización de apertura para el inicio de actividades es sustituida por una declaración responsable.

- En cuanto al procedimiento de evaluación de impacto ambiental, se modifican aspectos relativos a la presentación telemática del estudio de impacto ambiental y se establecen también los requisitos para la modificación de la declaración de impacto ambiental. 
- Los procedimientos sobre los distintos tipos de licencia de actividad clasificada también son desarrollados en aspectos muy variados (informes preceptivos, información pública). Se establecen reglas sobre la caducidad de la licencia y del otorgamiento de la licencia de obras mientras se tramita la licencia de actividad, y se sustituye la licencia de apertura para el inicio de la actividad por una declaración responsable.

- Se establece la previsión de una visita de inspección durante los primeros seis meses de funcionamiento de una instalación que haya obtenido una primera autorización ambiental integrada o a una nueva como consecuencia de la realización de una modificación sustancial.

- El anexo II enumera las instalaciones que dejan de estar sometidas a autorizaciones ambientales, siendo sustituidas estas por declaraciones responsables.

- El anexo III precisa las actividades que presentan riesgos para la salud de las personas y que precisan informe preceptivo y vinculante del Instituto de Salud Pública y Laboral de Navarra.

\section{La regulación de impuestos energéticos ambientales}

Mediante la Ley Foral 11/2015, de 18 de marzo, se han regulado en la Comunidad Foral de Navarra dos impuestos energéticos de contenido ambiental: el Impuesto sobre el Valor de la Producción de la Energía Eléctrica y el Impuesto sobre los Gases Fluorados de Efecto Invernadero. Especialmente, este último constituye un medio más para luchar contra el cambio climático, dado que se grava la emisión de un tipo de gases generadores del efecto invernadero.

Estos impuestos energéticos están regulados en el Estado en la Ley 15/2012, de 27 de diciembre, de medidas fiscales para la sostenibilidad energética, y en la Ley 16/2013, de 29 de octubre, por la que se establecen determinadas medidas en materia de fiscalidad medioambiental. En virtud de las competencias tributarias de Navarra, era necesaria una ley foral para la exacción de estos tributos en la Comunidad Foral. Las normas sustantivas y formales aplicables a ambos impuestos son de idéntico contenido a las establecidas por el Estado. Por eso, me limitaré a señalar la definición de estos impuestos y los supuestos en los que la exacción corresponde a Navarra. 
El Impuesto sobre el Valor de la Producción de la Energía Eléctrica es un tributo de carácter directo y naturaleza real que grava la realización de actividades de producción e incorporación al sistema eléctrico de energía eléctrica medida en barras de central, a través de cada una de las instalaciones de producción de energía eléctrica (art. 1.2). Su exacción corresponde a la Comunidad Foral cuando las instalaciones de producción de energía eléctrica radiquen en Navarra. Si la instalación está situada en territorio navarro y en territorio común, la exacción del impuesto corresponderá a la Administración competente para su autorización (art. 1.3).

El Impuesto sobre los Gases Fluorados de Efecto Invernadero es un tributo de naturaleza indirecta que recae sobre el consumo de aquellos productos comprendidos en su ámbito objetivo y grava, en fase única, el consumo de estos productos atendiendo al potencial de calentamiento atmosférico (art. 2.2). La exacción del mencionado Impuesto sobre los Gases Fluorados de Efecto Invernadero corresponderá a Navarra cuando los consumidores finales utilicen los productos objeto del impuesto en instalaciones, equipos o aparatos radicados en Navarra. Cuando los gases fluorados de efecto invernadero sean objeto de autoconsumo, la exacción corresponderá a la Comunidad Foral en el supuesto de que dicho autoconsumo se produzca en su territorio (art. 2.4).

\section{Relación de disposiciones}

— Ley Foral 11/2015, de 18 de marzo, por la que se regula el Impuesto sobre el Valor de la Producción de la Energía Eléctrica, el Impuesto sobre los Gases Fluorados de Efecto Invernadero y el Impuesto sobre los Depósitos en las Entidades de Crédito (BON, núm. 62, de 31 de marzo de 2015).

- Decreto Foral 105/2014, de 5 de noviembre, por el que se designa el Lugar de Importancia Comunitaria denominado "Belate" como Zona Especial de Conservación y se aprueba su Plan de Gestión (BON, núm. 245, de 17 de diciembre de 2014).

- Decreto Foral 117/2014, de 29 de diciembre por el que se designa el Lugar de Importancia Comunitaria denominado "Sierra de Aralar" como Zona Especial de Conservación y se aprueba su Plan de Gestión (BON, núm. 16, de 26 de enero de 2015).

- Orden Foral 448/2014, de 23 de diciembre, del Consejero de Desarrollo Rural, Medio Ambiente y Administración Local, por la que se aprueban las normas de desarrollo del Decreto Foral 93/2006, de 28 de diciembre, por el que se aprueba el 
Reglamento de desarrollo de la Ley Foral 4/2005, de 22 de marzo, de Intervención para la Protección Ambiental, a fin de incorporar medidas de agilización administrativa y simplificación procedimental, en la forma que se recoge en el anexo I de la presente Orden Foral (BON, núm. 27, de 10 de febrero de 2015).

— Orden Foral 42/2015, de 10 de febrero, del Consejero de Desarrollo de Rural, Medio Ambiente y Administración Local, por la que se aprueba la normativa específica que regirá la pesca en Navarra durante el año 2015, incluyendo determinadas medidas de control de poblaciones de especies exóticas invasoras (BON, núm. 37, de 24 de febrero de 2015).

- Orden Foral 82/2015, de 6 de marzo, por la que se aprueba el Programa de Inspección Ambiental de la Actividad Industrial de la Comunidad Foral de Navarra 2015 (BON, núm. 71, de 15 de abril de 2015).

- Orden Foral 110/2015, de 13 de abril, por la que se establecen las normas de la condicionalidad que deben cumplir los beneficiarios que reciban pagos directos, primas anuales de desarrollo rural, o pagos en virtud de determinados programas de apoyo al sector vitivinícola (BON, núm. 69, de 13 de abril de 2015).

- Resolución 211/2015, del Director General de Medio Ambiente y Agua, por la que se modifica el pliego de condiciones y el formulario técnico para la elaboración de los Planes de Ordenación Cinegética en Navarra, aprobados por Resolución 125/2012, de 26 de enero (BON, núm. 75, de 21 de abril de 2015). 